\title{
Analysis of ventilator-associated pneumonia infection route by genome macrorestriction-pulsed-field gel electrophoresis and its prevention with combined nursing strategies
}

\author{
XIAODONG WANG ${ }^{1}$, JUNPING WANG ${ }^{1}, \mathrm{JING} \mathrm{LI}^{2}$ and JING WANG ${ }^{1}$ \\ Departments of ${ }^{1}$ Pulmonary and Critical Care Medicine and ${ }^{2}$ Nursing, The First Affiliated Hospital of Zhengzhou University, \\ Zhengzhou, Henan 450052, P.R. China
}

Received February 24, 2014; Accepted September 10, 2014

DOI: $10.3892 /$ etm.2014.1994

\begin{abstract}
The aim of the present study was to explore the infection route of ventilator-associated pneumonia (VAP) and assess the effectiveness of a combined nursing strategy to prevent VAP in intensive care units. Bacteria from the gastric juice and drainage from the hypolarynx and lower respiratory tracts of patients with VAP were analyzed using genome macrorestriction-pulsed-field gel electrophoresis (GM-PFGE). A total of 124 patients with tracheal intubation were placed in the intervention group and were treated with a combined nursing strategy, comprising mosapride (gastric motility stimulant) administration and semi-reclining positioning. A total of 112 intubated patients were placed in the control group and received routine nursing care. The incidence rate of VAP, days of ventilation and mortality rate of patients were compared between the two groups. The GM-PFGE fingerprinting results of three strains of Pseudomonas aeruginosa from the gastric juice, subglottic secretion drainage and drainage of the lower respiratory tract in patients with VAP were similar across groups. The number of days spent on a ventilator by patients in the intervention group (7.37 \pm 5.32 days) was lower compared with that by patients in the control group (12.34 \pm 4.98 days) $(\mathrm{P}<0.05)$. The incidence rate of VAP was reduced from 40.81 to $21.25 \%$ following intervention with the combined nursing strategy $(\mathrm{P}<0.05)$; furthermore, the mortality rate of intubated patients in the intervention group was $29.46 \%$, a significant reduction compared with the $41.94 \%$ mortality rate observed in the control group $(\mathrm{P}<0.05)$. Gastroesophageal reflux (GER) was confirmed as one of the infection routes for VAP. The combined
\end{abstract}

Correspondence to: Dr Jing Wang, Department of Pulmonary and Critical Care Medicine, The First Affiliated Hospital of Zhengzhou University, 1 Jianshe Dong Road, Zhengzhou, Henan 450052, P.R. China

E-mail: jingwang0012013@gmail.com

Key words: ventilator-associated pneumonia, interventions, intensive care unit, genome macrorestriction-pulsed-field gel electrophoresis nursing strategy of gastric motility stimulant administration and the adoption of a semi-reclining position was effective in preventing VAP by reducing the occurrence of GER.

\section{Introduction}

Ventilator-associated pneumonia (VAP) is one of the most common hospital-acquired pneumonias occurring in intubated patients and remains a leading cause of morbidity and mortality of patients in intensive care units (ICUs). Ventilator dependence and long hospital stays increase the costs of hospitalization. A number of risk factors exist for VAP, with bacterial colonization of the gastric content with subsequent gastroesophageal reflux (GER) and aspiration into the airways being an important risk factor (1). There is currently no particular method of preventing VAP; however, there are several promising combined nursing strategies that are effective in preventing VAP, including education programs, oral care and the continuous control of endotracheal cuff pressure and microaspiration of gastric contents. Dodek et al demonstrated that appropriate positioning to reduce reflux, improving oral hygiene, may reduce the incidence of VAP (2). In the present study, evidence for a retrograde route of VAP transmission from the stomach to the oropharynx to the lower respiratory tract was examined. Genome macrorestriction-pulsed-field gel electrophoresis (GM-PFGE) was performed to accomplish this and to subsequently assess the effects of a combined intervention strategy, comparing the mean risk of VAP between the control and intervention groups.

\section{Materials and methods}

Diagnosis of VAP. At present, the definition of VAP is controversial as it is difficult to distinguish the condition from other usual pulmonary infections. According to the definition by the American Thoracic Society and the Infectious Diseases Society of America, VAP is considered to be pneumonia in patients that have received mechanical ventilation for $\geq 48 \mathrm{~h}$, characterized by the presence of a new or progressive infiltrate and signs of systemic infection (including temperature, blood cell count, changes in sputum characteristics and detection of the causative agent) (3). The condition can be divided into late-onset VAP (LOP) and early-onset VAP (EOP), depending 
on whether time spent on the ventilator was $>5$ or $<5$ days, respectively. In the present study, the clinical diagnosis criteria for VAP (positive quantitative endotracheal aspirate cultures and Clinical Pulmonary Infection Score $>6$ ) were based on the Hospital-Acquired Pneumonia Diagnosis and Treatment Guidelines by The Chinese Medical Association Branch of Respiratory Diseases (4).

A diagnosis of VAP (excluding certain associated lung diseases, including tuberculosis, lung cancer and atelectasis) was made if the condition of the patient met the following criteria: i) Lung infection following mechanical ventilation for $48 \mathrm{~h}$; ii) presence of infiltrates or new inflammatory pulmonary lesions following mechanical ventilation; and iii) lung consolidation and/or moist lung rales, as well as one of the following: a) Blood cells $>1.0 \times 10^{10} / 1$ or $<4 \times 10^{9} / 1$, with or without nuclear transfer; b) fever (body temperature $>37.5^{\circ} \mathrm{C}$ ) with a large number of purulent respiratory secretions; or c) new pathogenic bacteria isolated from bronchial secretions.

Genotyping by GM-PFGE. Respiratory secretions from four patients with VAP [randomly selected from the ICU of the First Affiliated Hospital of Zhengzhou University (Zhengzhou, China)] were collected using protected specimen brushes once every other day for quantitative bacterial culture. The bacterial stains that antimicrobial susceptibility testing showed to be undifferentiated were cultured by centrifugation in lysogeny broth (Beijing Solarbio Science \& Technology Co., Ltd, Beijing, China) at $37^{\circ} \mathrm{C}$ and $4.48 \times \mathrm{g}$ to a concentration of $10^{\circ} / \mathrm{ml}$ after $18 \mathrm{~h}$. The cells were suspended in $100 \mu \mathrm{l}$ buffer $(0.1 \mathrm{~mol} / \mathrm{l}$ EDTA, pH 8.0; $0.01 \mathrm{~mol} / 1$ Tris-Cl, $\mathrm{pH} 7.6$; and $1 \mathrm{~mol} / \mathrm{l} \mathrm{NaCl})$, mixed with $100 \mu$ l low-melting point agarose (Sangon Biotech Co., Ltd., Shanghai, China) and molded into plugs at $4^{\circ} \mathrm{C}$. Following congealing of the agar gel, the cells were lysed with lysis buffer at $37^{\circ} \mathrm{C}$ for $2 \mathrm{~h}$ and washed with Sodium Chloride-Tris-EDTA (STE) buffer $(0.1 \mathrm{~mol} / 1 \mathrm{NaCl} ; 10 \mathrm{mmol} / 1$ Tris-Cl, $\mathrm{pH} 8.0$; and $1 \mathrm{mmol} / 1$ EDTA, PH 8.0), followed by enzymolysis with proteinase K (1 mg/ml; Sangon Biotech Co., Ltd.) for 16-20 h. The lysed cells were subsequently treated with $2 \mathrm{mmol} / \mathrm{l}$ phenylmethanesulfonyl fluoride (Beijing Cowin Bioscience Co., Ltd., Beijing, China) for $45 \mathrm{~min}$; this treatment was repeated once more, prior to washing three times with STE buffer and mixing at room temperature with a buffer containing 50 units SpeI restriction enzyme (Beijing Cowin Bioscience Co., Ltd.). After $18 \mathrm{~h}$ at $37^{\circ} \mathrm{C}$, electrophoresis was performed using GenePath (Bio-Rad, Hercules, CA, USA) at a field strength of $6 \mathrm{~V} / \mathrm{cm}$, at $14^{\circ} \mathrm{C}$ for $20 \mathrm{~h}$. The pulse times were between 5 and $35 \mathrm{sec}$. The gel was then stained with ethidium bromide $(0.5 \mu \mathrm{g} / \mathrm{ml}$; HaoSen Co., Jiangsu, China) for $1 \mathrm{~h}$ and washed for $1 \mathrm{~h}$ with $\mathrm{ddH}_{2} \mathrm{O}$. Finally, the gel was observed under a $302 \mathrm{~nm}$ ultraviolet light and images were captured. Fragment patterns were compared according to the criteria set out by Tenover et al (5).

\section{Study population}

Inclusion and exclusion criteria. The inclusion criteria for the patients observed in the present study were as follows: i) $>60$ years old and ii) receiving mechanical ventilation. The exclusion criteria comprised: i) a critical condition that could cause mortality within $48 \mathrm{~h}$; ii) mechanical ventilation for $<48 \mathrm{~h}$; and iii) pulmonary infection.
Intervention group. The 124 intubated patients (intervention group) were treated at the ICU of the First Affiliated Hospital of Zhengzhou University between January 2011 and May 2013. The intervention treatment included the administration of a gastric motility stimulant and the adoption of a semi-reclining position. Mosapride citrate tablets $(5 \mathrm{mg} / \mathrm{tablet}$; Lunan Company, Shandong, China) at a dosage of $5 \mathrm{mg} / \mathrm{admin}-$ istration, three times a day, were selected as the gastric motility stimulant. The stimulant was used continuously until the study end-point or unless severe diarrhea (loose bowel movements three times in one day) occurred.

The semi-reclining position was used as a treatment if there were no tolerance issues or anti-semi-reclining indicators (including use of a vascular active drug, refractory shock following hypervolemic therapy inefficiency, or neurosurgery or abdominal surgery within the previous seven days). The beds of able patients were positioned at an angle of $30-45^{\circ}$ to maintain these patients in a semi-reclining repose; for those patients that exhibited tolerance issues, a semi-reclining position was maintained during nasogastric feeding (including gastrointestinal injections), as well as for $2 \mathrm{~h}$ after feeding.

Control group. A total of 112 intubated patients received traditional nursing in the ICU of the First Affiliated Hospital of Zhengzhou University between January 2009 and December 2010. The patients were not administered any gastric motility stimulants during the treatment period.

All other procedures in the treatment of the two groups of patients were consistent and included the following: i) Monitoring of intra-cuff pressure every $4 \mathrm{~h}$ and maintenance at $>20 \mathrm{mmHg}$, as well as the continuous aspiration of subglottic secretion, sputum smear, Gram staining and aerobic cultivation twice per week; ii) administration of sucralfate tablets; and iii) no use of $\mathrm{H}_{2}$ receptor antagonists and antacids unless bleeding of the stress ulcers occurred. In addition, Acute Physiology and Chronic Health Evaluation II (APACHE II) score of all patients were evaluated. APACHE II was an indicator of illness severity, which was determined using the worst value obtained during the initial $24 \mathrm{~h}$ following ICU admission, as well as on the day of VAP diagnosis $(6,7)$.

Written informed consent was obtained from all patients prior to their involvement in the study. The study was approved by the Life Sciences Institutional Review Board of Zhengzhou University.

Observation indices. A number of observations were recorded, including baseline indices (characteristics of the patients prior to treatment), length of hospital stay and number of days spent on the ventilator. In addition, GER was monitored in all the patients. The observation was concluded when one of the following occurred: Mortality, extubation or diagnosis of VAP with a one month follow-up to confirm whether the cause of the patient's mortality was associated with VAP. The incidence rate of VAP referred to the number of VAP episodes per 1,000 ventilator-days. Mortality rates for the patients with and without VAP were calculated and compared between the two groups of patients.

Statistical analysis. All statistical data were analyzed using SPSS 12.0 software (SPSS, Inc, Chicago, IL, USA). Rate data 
Table I. Basic participant information.

\begin{tabular}{lccc}
\hline & \multicolumn{3}{c}{ Group } \\
\cline { 2 - 3 } Characteristic & Control & Intervention & \\
\hline $\mathrm{n}$ & 112 & 124 & - \\
Age in years, mean \pm SD & $70.63 \pm 6.31$ & $71.06 \pm 5.74$ & 0.851 \\
Gender, $\mathrm{n}$ male/n female & $67 / 57$ & $59 / 53$ & 0.764 \\
APACHE II score, mean $\pm \mathrm{SD}$ & $22.84 \pm 5.75$ & $24.84 \pm 4.96$ & 0.672 \\
\hline
\end{tabular}

APACHE II, Acute Physiology and Chronic Health Evaluation II; SD, standard deviation.

Table II. Comparison of several indexes between the control and intervention groups.

\begin{tabular}{lccr}
\hline & & Group & \\
\cline { 2 - 3 } Indexes & Control & Intervention & P-value \\
\hline VAP, n (EOP/LOP) & $84(26 / 58)$ & $43(11 / 23)$ & 0.882 \\
Ventilator-days, mean \pm SD & $12.34 \pm 4.98$ & $7.37 \pm 5.32$ & 21.25 \\
Incidence of VAP,$\% 0$ & 40.81 & 29.46 & $<0.050$ \\
Mortality rate, $\%$ & & 47.06 & $<0.050$ \\
Intubated patients & 41.94 & 0.252 \\
Patients with VAP & 35.71 & & \\
\hline
\end{tabular}

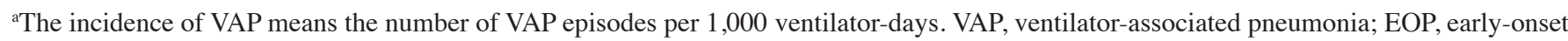
VAP; LOP, late-onset VAP; SD, standard deviation.

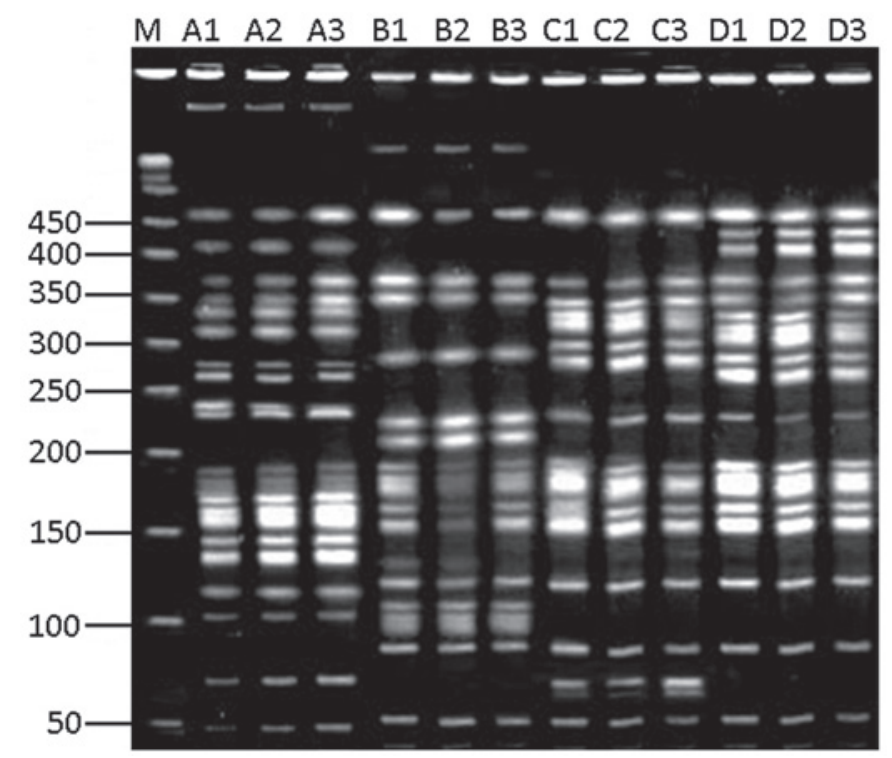

Figure 1. Genome macrorestriction-pulsed-field gel electrophoresis for 12 strains of Pseudomonas aeruginosa from four patients with VAP. M indicates the molecular size markers ( $\lambda$ ladder DNA). The letters A, B, C and D indicate the four patients with VAP. The numbers refer to the origin of the pathogens: 1, gastric juice; 2, subglottic secretion drainage and 3 drainage from the lower respiratory tract. VAP, ventilator-associated pneumonia.

were calculated using a $\chi^{2}$ test. Significant differences between the two groups with one variant were determined using the Student' t-test. A two-tailed value of $\mathrm{P}<0.05$ was considered to indicate a statistically significant difference.

\section{Results}

GM-PFGE fingerprinting. The primary pathogen, Pseudomonas aeruginosa, was selected for genotyping 
using GM-PFGE. GM-PFGE fingerprinting indicated that the $P$. aeruginosa from the gastric juice, subglottic secretion drainage and drainage of the lower respiratory tract were similar in each patient with VAP (Fig. 1). However, while the strains were consistent across locations in a single patient, the strains observed across the four patients with VAP differed.

Basic participant information. All patients in the intervention and control groups were selected strictly in accordance with the inclusion and exclusion criteria. The age, gender and basic data of the patients were normalized between the two groups. Furthermore, all patients were treated in the same hospital and the number of patients who were from medical or surgical departments was also normalized across the groups (Table I).

Etiology of VAP. The most commonly detected bacteria were P. aeruginosa (29.42\%), Acinetobacter baumannii (10.85\%), Staphylococcus aureus $(7.48 \%)$ and Stenotrophomonas maltophilia (7.32\%). The number of patients with VAP infected with two or more bacteria was $55.81 \%$. The primary pathogens identified in patients with EOP were Gram-positive bacteria (59.27\%), including S. aureus, while Gram-negative bacteria (including $P$. aeruginosa and $A$. baumannii) were most frequently identified in patients with LOP (70.8\%).

Effect of intervention. Table II shows that the combined nursing strategy described in the present study was able to decrease the number of days spent on a ventilator, the incidence rate of VAP and the mortality rate of the intubated patients (all $\mathrm{P}<0.05$ ); however, no significant difference in the ratio of EOP/LOP was found between the two groups. The mortality rate from VAP was not reduced by these nursing measures once it had occurred.

\section{Discussion}

PFGE has been used as the gold standard for the genotyping of bacteria, which can be a powerful tool for the study of nosocomial infections. There is debate as to whether a retrograde route of transmission from the stomach to oropharynx to lower respiratory tract contributes to VAP. In the present study, GM-PFGE fingerprinting results demonstrated that this route does exist in patients with VAP. This result was consistent with that of a previous study (8) and may be the reason why the interventions in the present study were effective in the prevention of VAP. Grap et al (9) observed that an early, single application of chlorhexidine significantly reduced the occurrence of VAP in trauma patients. The results of the present study indicate that this may have been due to the chlorhexidine blocking the infection route from the stomach to respiratory tract, at least to a certain extent.

In the present study, the results of GM-PFGE genotyping indicated that the $P$. aeruginosa populations at different drainage locations in each patient were consistent. A previous animal study involving New Zealand white rabbits indicated that bacteria from the gastrointestinal tracts of the rabbits were not the main sources of EOP, but may have contributed to the development of LOP (10). Consequently, the prevention of VAP through inhibition of the gastroesophageal reflux (GER) may be effective.
Numerous studies have been performed to investigate the prevention of VAP, and several strategies have been proven to be effective preventative measures, including educational intervention $(11,12)$, sufficient and professional oral care (13-15) and the use of specific equipment, such as heat and moisture exchange filters (16) or silver-coated endotracheal tubes (17). None of these measures, however, should be used as an isolated intervention.

The combined strategy used in the present study, which focused predominantly on body position and the inhibition of the occurrence of GER, revealed that a semi-reclining position and the use of mosapride (an inhibitor of GER) were able to decrease the incidence rate of VAP from 40.81 to $21.25 \%$ $(\mathrm{P}<0.05)$. The number of days spent on a ventilator was reduced by almost five days in the intervention group $(\mathrm{P}<0.05)$, and the mortality rate of intubated patients decreased from 41.94 to $29.46 \%(\mathrm{P}<0.05)$. However, the interventions had no effect on the mortality rate following the occurrence of VAP, demonstrating that the effectiveness of these interventions lies entirely in preventing VAP onset. These results were consistent with those of a previously published study (18). An animal model of mechanical ventilation using healthy New Zealand white rabbits demonstrated that drugs promoting gastrointestinal motility (mosapride citrate) were useful in reducing the incidence rate of VAP caused by bacteria from the digestive tract (10).

As the development of VAP is a complicated process, its overall prevention requires numerous stages, courses and methods. Further studies are required to investigate the infection route from the stomach to the respiratory tract in patients with VAP, and more effective and personalized strategies for reducing episodes of VAP should be utilized.

\section{Acknowledgements}

This study was supported by the 'Project of Medical Science and Technology Research of Henan Province' (no. 201203032) and the 'Project of Science and Technology Research of Zhengzhou City' (no. 0910SGYS33389-13).

\section{References}

1. Abdel-Gawad TA, El-Hodhod MA, Ibrahim HM and Michael YW: Gastroesophageal reflux in mechanically ventilated pediatric patients and its relation to ventilator-associated pneumonia. Crit Care 13: R164, 2009.

2. Dodek P, Keenan S, Cook D, et al; Canadian Critical Care Trials Group; Canadian Critical Care Society: Evidence-based clinical practice guideline for the prevention of ventilator-associated pneumonia. Ann Intern Med 141: 305-313, 2004.

3. American Thoracic Society; Infectious Diseases Society of America: Guidelines for the management of adults with hospital-acquired, ventilator-associated, and healthcare-associated pneumonia. Am J Respir Crit Care Med 171: 388-416, 2005.

4. Xue XY, Gao ZC, Zhu JH, Xu Y and Li X: Clinical evaluation of chinese guidelines for community-acquired pneumonia. Beijing Da Xue Xue Bao 38: 276-279, 2006 (In Chinese).

5. Tenover FC, Arbeit RD, Goering RV, et al: Interpreting chromosomal DNA restriction patterns produced by pulsed-field gel electrophoresis: criteria for bacterial strain typing. J Clin Microbiol 33: 2233-2239, 1995.

6. Gursel G and Demirtas S: Value of APACHE II, SOFA and CPIS scores in predicting prognosis in patients with ventilator-associated pneumonia. Respiration 73: 503-508, 2006. 
7. Elwakil MA: APACHE II scoring and antibiotics significance against VAP associated risks. Pak J Biol Sci 14: 1036-1037, 2011.

8. Schleder BJ: Taking charge of hospital-acquired pneumonia. Nurse Pract 29: 50-53, 2004.

9. Grap MJ, Munro CL, Hamilton VA, Elswick RK Jr, Sessler CN and Ward KR: Early, single chlorhexidine application reduces ventilator-associated pneumonia in trauma patients. Heart Lung 40: e115-e122, 2011.

10. Liu Q, He LX, Hu BJ, et al: The role of gastro-intestinal tract microorganisms in the development of ventilator-associated pneumonia: an experimental study. Zhonghua Jie He He Hu X Za Zhi 31: 509-512, 2008.

11. Kellie SP, Scott MJ, Cavallazzi R, et al: Procedural and educational interventions to reduce ventilator-associated pneumonia rate and central line-associated blood stream infection rate. J Intensive Care Med 29: 165-174, 2014.

12. Jansson $M$, Kääriäinen $M$ and Kyngäs $H$ : Effectiveness of educational programmes in preventing ventilator-associated pneumonia: a systematic review. J Hosp Infect 84: 206-214, 2013.
13. Al-Tawfiq JA, Amalraj A and Memish ZA: Reduction and surveillance of device-associated infections in adult intensive care units at a Saudi Arabian hospital, 2004-2011. Int J Infect Dis 17: e1207-e1211, 2013.

14. Scannapieco FA and Binkley CJ: Modest reduction in risk for ventilator-associated pneumonia in critically ill patients receiving mechanical ventilation following topical oral chlorhexidine. J Evid Based Dent Pract 12: 15-17, 2012.

15. Zurmehly J: Oral care education in the prevention of ventilator-associated pneumonia: quality patient outcomes in the intensive care unit. J Contin Educ Nurs 44: 67-75, 2013.

16. Auxiliadora-Martins M, Menegueti MG, Nicolini EA, et al: Effect of heat and moisture exchangers on the prevention of ventilator-associated pneumonia in critically ill patients. Braz J Med Biol Res 45: 1295-1300, 2012.

17. Kane T and Claman F: Silver tube coatings in pneumonia prevention. Nurs Times 108: 21-23, 2012.

18. Li Bassi G and Torres A: Ventilator-associated pneumonia: role of positioning. Curr Opin Crit Care 17: 57-63, 2011. 